\title{
First insights on value-based healthcare of elders using ICHOM older person standard set reporting
}

Wei-Ju Lee ${ }^{1,2,3}$, Li-Ning Peng ${ }^{1,2,4}$, Chi-Hung Lin' ${ }^{1}$, Shinn-Zong Lin ${ }^{5}$, Ching-Hui Loh' ${ }^{6}$, Sheng-Lun Kao ${ }^{6}$, Tzu-Shing Hung ${ }^{7}$, Chia-Yun Chang ${ }^{8}$, Chun-Feng Huang ${ }^{9}$, Ting-Ching Tang ${ }^{10}$ and Liang-Kung Chen ${ }^{1,2,4^{*}}$

\begin{abstract}
Background: Clinical guidelines for specific conditions fragment care provision for elders. The International Consortium for Health Outcomes Measurement (ICHOM) has developed a global standard set of outcome measures for comprehensive assessment of older persons. The goal of this study was to report value-based health metrics in Taiwan using this ICHOM toolset.

Methods: The cross-sectional study of baseline data excerpted from a prospective longitudinal cohort, which recruited people $\geq 65$ years old with $\geq 3$ chronic medical conditions between July and December 2018. All participants received measurements of physical performance, anthropometric characteristics, health-related behaviors, Charlson Comorbidity Index, and Montreal Cognitive Assessment. The ICHOM toolset comprises three tiers: 1 includes frailty and having chosen a preferred place of death; 2 includes polypharmacy, falls, and participation in decision-making; and 3 includes loneliness, activities of daily living, pain, depression, and walking speed. These items were converted into a 0-10 point value-based healthcare score, with high value-based health status defined as $\geq 8 / 10$ points.
\end{abstract}

Results: Frequencies of individual ICHOM indicators were: frail 11.7\%, chose preferred place of death $14.4 \%$, polypharmacy $31.5 \%$, fell $17.1 \%$, participated in decision-making $81.6 \%$, loneliness $26.8 \%$, limited activities of daily living 22.4\%, pain 10.4\%, depressed mood 13.0\%, and slowness 38.5\%. People with high disease burden (OR 0.40, $95 \% \mathrm{Cl} 0.21-0.76, p=0.005$ ) or cognitive impairment ( $\mathrm{OR} 0.49,95 \% \mathrm{Cl} 0.27-0.87, p=0.014$ ) were less likely to have high value-based healthcare status.

Conclusions: The ICHOM Standard Set Older Person health outcome measures provide an opportunity to shift from a disease-centric medical paradigm to whole person-focused goals. This study identified advanced age, chronic disease burden and cognitive impairment as important barriers to achieving high value-based healthcare status.

Keywords: International consortium for health outcomes measurement, Elder adult, Age, Value, Healthcare

\footnotetext{
* Correspondence: Ikchen2@vghtpe.gov.tw

${ }^{1}$ Aging and Health Research Center, National Yang Ming University, Taipei,

Taiwan

2Department of Geriatric Medicine, School of Medicine, National Yang Ming University, Taipei, Taiwan

Full list of author information is available at the end of the article
}

C C The Author(s). 2020 Open Access This article is licensed under a Creative Commons Attribution 4.0 International License, which permits use, sharing, adaptation, distribution and reproduction in any medium or format, as long as you give appropriate credit to the original author(s) and the source, provide a link to the Creative Commons licence, and indicate if changes were made. The images or other third party material in this article are included in the article's Creative Commons licence, unless indicated otherwise in a credit line to the material. If material is not included in the article's Creative Commons licence and your intended use is not permitted by statutory regulation or exceeds the permitted use, you will need to obtain permission directly from the copyright holder. To view a copy of this licence, visit http://creativecommons.org/licenses/by/4.0/ The Creative Commons Public Domain Dedication waiver (http://creativecommons.org/publicdomain/zero/1.0/) applies to the data made available in this article, unless otherwise stated in a credit line to the data. 


\section{Background}

A single-disease model has prevailed over centuries of medical progress, but the era of population aging brings major challenges of managing multimorbidity in older adults that threaten to fragment care provision by necessitating multiple assessments and treatments [1]. Healthcare systems will be increasingly burdened by fragmented services, higher service volumes and escalating associated medical costs, and are hence transitioning from volume-based to value-based provision that emphasizes quality, expenditure and patient experience [2]. Consequently, the question of how best to measure healthcare quality and outcomes has become a research priority. Specific models of value-based healthcare, such as pay-for-performance, have shown effectiveness in certain diseases or chronic conditions but not overall [3]. Moreover, prevalent chronic comorbidities in older adults [1] make it hard to measure variations in health outcomes. More 'function-centric' aging medicine is crucial to handling the diversity and complexity of health care for older people and promoting healthy aging [4].
The International Consortium for Health Outcomes Measurement (ICHOM) has initiated an ambitious project to develop value-based health metrics for specific groups of people rather than discrete diseases/conditions. To establish a standard health outcome set and improve care and quality pathways for older adults, the ICHOM convened a global expert consensus panel to formulate evidence-based outcome measurement tools [5]. Without such tools, it is difficult for policymakers and health professionals to choose interventions effective in improving care quality [6]. The ICHOM Standard Set of health outcomes for older persons will be conductive to supplanting piecemeal care of older persons with a more holistic approach. ICHOM standard set for older adults might provide an operative definition for high value-based healthcare services and an opportunity for healthcare providers and policy makers to examine and refine services provisions.

Since the ICHOM Standard Set Older Person was published in 2018, important questions remain. For example, which patient subgroups require comprehensive

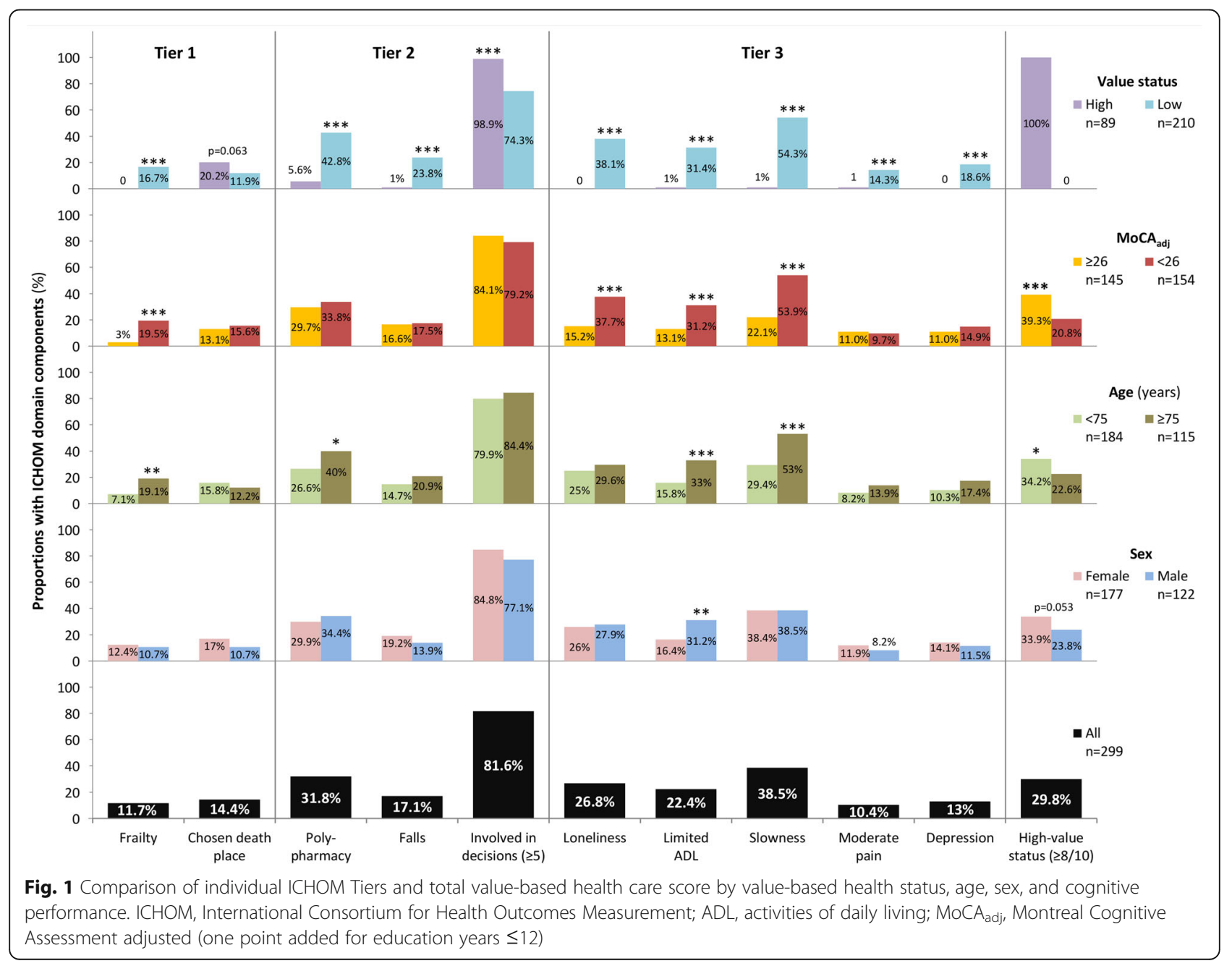


Table 1 ICHOM Standard Set Older Person outcome measures by value-based health status

\begin{tabular}{|c|c|c|c|c|}
\hline \multirow{2}{*}{$\begin{array}{l}\text { Data values show mean } \pm \text { standard deviation or number } \\
\text { (percent) }\end{array}$} & \multirow{2}{*}{$\begin{array}{l}\text { Entire } \\
\text { cohort }\end{array}$} & \multicolumn{2}{|c|}{ Value-based health status } & \multirow[t]{2}{*}{$p$} \\
\hline & & $\operatorname{Low}(<8 / 10)$ & High $(\geq 8 / 10)$ & \\
\hline Number & 299 & 210 & 89 & \\
\hline \multicolumn{5}{|l|}{ ICHOM Standard Set Older Person Tier 1} \\
\hline Clinical frailty scale & $2.7 \pm 0.9$ & $2.8 \pm 1.0$ & $2.3 \pm 0.7$ & $<0.001$ \\
\hline Frail & $35(11.7)$ & $35(16.7)$ & $0(0.0)$ & $<0.001$ \\
\hline Preferred place of death chosen & $43(14.4)$ & $25(11.9)$ & $18(20.2)$ & 0.061 \\
\hline Do Not Resuscitate signed & $21(7.0)$ & $11(5.2)$ & $10(11.2)$ & 0.064 \\
\hline \multicolumn{5}{|l|}{ ICHOM Standard Set Older Person Tier 2} \\
\hline Number of drugs & $3.6 \pm 2.7$ & $4.3 \pm 2.7$ & $2.0 \pm 1.7$ & $<0.001$ \\
\hline Polypharmacy ( $\geq 5$ concurrent drugs) & $95(31.8)$ & $90(42.8)$ & $5(5.6)$ & $<0.001$ \\
\hline Number of adverse drug events in past 12 months & $0.0 \pm 0.1$ & $0.0 \pm 0.2$ & $0.0 \pm 0.0$ & 0.180 \\
\hline Episodes of discomfort after medications in past 12 months & $0.0 \pm 0.1$ & $0.0 \pm 0.2$ & $0.0 \pm 0.0$ & 0.103 \\
\hline Fell in past 12 months & $51(17.1)$ & $50(23.8)$ & $1(1.1)$ & $<0.001$ \\
\hline Number of falls in past 12 months & $0.2 \pm 0.6$ & $0.3 \pm 0.7$ & $0.0 \pm 0.1$ & $<0.001$ \\
\hline Hospital admissions in past 12 months & $0.2 \pm 0.4$ & $0.2 \pm 0.5$ & $0.1 \pm 0.3$ & 0.004 \\
\hline Length of hospital stay (days) & $1.1 \pm 3.7$ & $1.4 \pm 4.3$ & $0.5 \pm 1.8$ & 0.009 \\
\hline Able to cope with own health & $276(92.3)$ & $187(89.1)$ & $89(100.0)$ & 0.001 \\
\hline Participate in care decision-making & $276(92.3)$ & $188(89.5)$ & $88(98.9)$ & 0.006 \\
\hline Treated with dignity and respect & $289(96.7)$ & $200(95.2)$ & $89(100.0)$ & 0.036 \\
\hline Received coordinated care & $270(90.3)$ & $181(86.2)$ & $89(100.0)$ & $<0.001$ \\
\hline Discharged to place of choice & $296(99.0)$ & $207(98.6)$ & $89(100.0)$ & 0.257 \\
\hline Overall participation in decision-making & $4.7 \pm 0.7$ & $4.6 \pm 0.9$ & $5.0 \pm 0.1$ & $<0.001$ \\
\hline High participation $(\geq 5)$ & $244(81.6)$ & $156(74.3)$ & $88(98.9)$ & $<0.001$ \\
\hline \multicolumn{5}{|l|}{ ICHOM Standard Set Older Person Tier 3} \\
\hline UCLA Loneliness Scale & $31.0 \pm 10.0$ & $33.4 \pm 11.0$ & $25.3 \pm 3.0$ & $<0.001$ \\
\hline Loneliness & $80(26.8)$ & $80(38.1)$ & $0(0.0)$ & $<0.001$ \\
\hline Activities of daily living & $7.4 \pm 1.4$ & $7.2 \pm 1.6$ & $8.0 \pm 0.3$ & $<0.001$ \\
\hline Any limitation of activities of daily living & $67(22.4)$ & $66(31.4)$ & $1(1.1)$ & $<0.001$ \\
\hline Walking speed (m/s) & $0.9 \pm 0.3$ & $0.8 \pm 0.3$ & $1.1 \pm 0.2$ & $<0.001$ \\
\hline Slowness (6-m walk < $0.8 \mathrm{~m} / \mathrm{s}$ ) & $115(38.5)$ & $114(54.3)$ & $1(1.1)$ & $<0.001$ \\
\hline Moderate pain & $31(10.4)$ & $30(14.3)$ & $1(1.1)$ & $<0.001$ \\
\hline Depression & $39(13.0)$ & 39 (18.6) & $0(0.0)$ & $<0.001$ \\
\hline Value-based healthcare score & $7.2 \pm 1.8$ & $6.5 \pm 1.6$ & $9.1 \pm 0.3$ & $<0.001$ \\
\hline High value-based healthcare & 89 (29.8) & 0 & $89(100)$ & $<0.001$ \\
\hline
\end{tabular}

ICHOM International Consortium for Health Outcomes Measurement, UCLA University of California, Los Angeles

assessments to evaluate their needs? Reporting these health metrics is the first step towards pragmatic application of this tool. This study explored the application of the ICHOM Standard Set for Older Persons health status among older multi-morbid community-dwelling adults in Taiwan.

\section{Methods}

\section{Participants and study design}

This cross-sectional study recruited older multimorbid community-living adults in New Taipei City, Yi-Lan
County, and Hualien County, Taiwan, between July and December 2018. The inclusion criteria were: age $\geq 65$ years and $\geq 3$ chronic medical conditions. The study excluded people who: were unable to communicate adequately with study staff; had malignant tumors undergoing active chemotherapy; with life expectancy $<12$ months; were institutionalized. Supplementary figure 1 showed details of recruitment process.

This study was designed and conducted in accordance with the principles of the Declaration of Helsinki. The 
Table 2 Demographic and health-related characteristics by value-based health status

\begin{tabular}{|c|c|c|c|c|}
\hline \multirow{2}{*}{$\begin{array}{l}\text { Data values show mean } \pm \text { standard deviation or } \\
\text { number (percent) }\end{array}$} & \multirow{2}{*}{$\begin{array}{l}\text { Entire } \\
\text { cohort }\end{array}$} & \multicolumn{2}{|c|}{ Value-based health status } & \multirow[t]{2}{*}{$p$} \\
\hline & & $\operatorname{Low}(<8 / 10)$ & $\operatorname{High}(\geq 8 / 10)$ & \\
\hline \multicolumn{5}{|l|}{ Demographics and health-related factors } \\
\hline Number & 299 & 210 & 89 & \\
\hline Age (years) & $73.3 \pm 6.6$ & $74.0 \pm 6.9$ & $71.5 \pm 5.7$ & 0.002 \\
\hline Male & $122(40.8)$ & $93(44.3)$ & $29(32.6)$ & 0.060 \\
\hline Education (years) & $7.6 \pm 4.7$ & $7.3 \pm 4.7$ & $8.3 \pm 4.6$ & 0.063 \\
\hline Smoke tobacco & $44(14.7)$ & $36(17.1)$ & $8(9.0)$ & 0.069 \\
\hline Drink alcohol & $37(12.4)$ & $27(12.9)$ & $10(11.2)$ & 0.697 \\
\hline Exercise & $51(17.1)$ & $34(16.2)$ & $17(19.1)$ & 0.541 \\
\hline Montreal Cognitive Assessment (adjusted) ${ }^{\mathrm{a}}$ & $23.8 \pm 5.6$ & $22.8 \pm 5.9$ & $26.2 \pm 3.8$ & $<0.001$ \\
\hline Montreal Cognitive Assessment (adjusted) ${ }^{a}<26$ & $154(51.5)$ & $122(58.1)$ & $32(36.0)$ & $<0.001$ \\
\hline Charlson Comorbidity Index & $1.1 \pm 1.0$ & $1.3 \pm 1.1$ & $0.9 \pm 1.0$ & 0.002 \\
\hline Charlson Comorbidity Index $\geq 2$ & $90(30.1)$ & $74(35.2)$ & $16(18.0)$ & 0.003 \\
\hline Body mass index & $25.3 \pm 3.6$ & $25.2 \pm 3.8$ & $25.3 \pm 3.1$ & 0.797 \\
\hline
\end{tabular}

${ }^{\mathrm{a}}$ One point added for education year12

Institutional Review Board of National Yang-Ming University approved the protocol (YM107042F). All participants provided fully informed written consent. The design and reporting format follow STROBE guidelines [7].

\section{Value-based health metrics}

The ICHOM Standard Set Older Person comprises three tiers (Supplementary Table 1) [5]. Absent valid Taiwanese versions of 4-item screening Zarit Burden interview- measured carer burden- and the Adult Social Care Outcomes Toolkit -measured autonomy and control- mitigated their applications in this study. Mortality was excluded because of present baseline data. Tier 1, achieved or retained health status, includes: all cause survival; death in a chosen place; and frailty. Participants were asked whether they had expressed a preferred place to die, and frailty was defined as clinical frailty scale $\geq 4$ [8].

Tier 2, treatment burden and complications, includes: falls in the last 12 months; polypharmacy with $\geq 5$ drugs [9]; and participation in decision-making, which comprised confidence in ability to manage their own health, discussion and planning of care, being treated with dignity and respect, coordination of care, and discharge to a chosen place. People in whom of these all components were affirmed were classed as having high participation in decision-making.

Tier 3, long-term consequences of care management and health sustainability, includes: loneliness, defined as $\geq 35$ points on the University of California, Los Angeles (UCLA) loneliness scale [10, 11]; limitation of daily activities (disability), defined as Lawton instrumental activities of daily living scale $<8$ (most independent) [12]; 6-m walk speed at usual pace, with $<0.8 \mathrm{~m} / \mathrm{s}$ defined as slowness [13]; pain and emotional health measured by the Short-
Form Health Survey (SF-36), with pain affecting activities of daily living considered pain, and the criterion for depression being $\geq 5 / 9$ SF-36 depressive symptoms [14].

Based on items in Tiers 1, 2 and 3 (Supplementary Table 1), a score ranging from 0 to 10 was derived to represent the value-based health status of each individual; a highest tertile score of $\geq 8 / 10$ was defined as high value-based health status.

\section{Other variables}

Physical performance, anthropometric characteristics, and health-related behaviors of all participants were recorded. Any tobacco or alcohol use in the last 6 months was classed as smoking or drinking, respectively. Exercise was defined as fitness activity for $\geq 30 \mathrm{~min}$ at least thrice weekly. Blood pressure, height and body weight were measured by standard procedures; body mass index was calculated as weight in kilograms, divided by height in meters squared $\left(\mathrm{kg} / \mathrm{m}^{2}\right)$. All participants were asked whether they had signed a Do Not Resuscitate order, which is an official agreement registered on national health insurance cards. Cognitive function was measured using The Montreal Cognitive Assessment (MoCA), adjusted by adding one point for those educated for $\leq 12$ years $\left(\mathrm{MoCA}_{\mathrm{adj}}\right)$; $\mathrm{MoCA}_{\mathrm{adj}} \geq 26$ constituted normal cognitive function [15]. Charlson Comorbidity Index quantified disease burden and comorbidity burdens, with high burden defined as a score of $\geq 2$ [16].

\section{Statistical analysis}

All analyses were performed with the SAS statistical package, version 9.4 (SAS Institute, Inc., Cary, NC, USA). A two-sided $p$-value $<0.05$ was considered statistically significant. Numerical variables were expressed as 
Table 3 Demographic data and ICHOM Standard Set Older Person outcome measures by sex, age, and cognitive performance status

\begin{tabular}{|c|c|c|c|c|c|c|c|c|c|}
\hline \multirow{2}{*}{$\begin{array}{l}\text { Data values show mean } \pm \text { standard deviation or } \\
\text { number (percent) }\end{array}$} & \multicolumn{3}{|l|}{ Sex } & \multicolumn{3}{|l|}{ Age (years) } & \multicolumn{3}{|l|}{ MoCA adj } \\
\hline & Female & Male & $p$ & $<75$ & $\geq 75$ & $p$ & $\geq 26$ & $<26$ & $p$ \\
\hline \multicolumn{10}{|l|}{ Demographics and health-related factors } \\
\hline Number & 177 & 122 & & 184 & 115 & & 145 & 154 & \\
\hline Age (years) & $73.3 \pm 6.3$ & $73.3 \pm 7.0$ & 0.957 & $68.9 \pm 2.7$ & $80.3 \pm 4.6$ & $<0.001$ & $71.3 \pm 5.5$ & $75.1 \pm 7.0$ & $<0.001$ \\
\hline Male & $0(0)$ & $122(100)$ & $<0.001$ & $75(40.8)$ & $47(40.9)$ & 0.985 & $57(39.3)$ & $65(42.2)$ & 0.61 \\
\hline Education (years) & $6.9 \pm 4.4$ & $8.8 \pm 4.8$ & 0.001 & $8.3 \pm 4.3$ & $6.5 \pm 5.0$ & 0.001 & $9.3 \pm 4.0$ & $6.0 \pm 4.7$ & $<0.001$ \\
\hline Smoke tobacco & $6(3.4)$ & $38(31.2)$ & $<0.001$ & $29(15.8)$ & $15(13.0)$ & 0.519 & $21(14.5)$ & $23(14.9)$ & 0.912 \\
\hline Drink alcohol & $7(4.0)$ & $30(24.6)$ & $<0.001$ & $25(13.6)$ & $12(10.4)$ & 0.421 & $24(16.6)$ & $13(8.4)$ & 0.033 \\
\hline Exercise ( $\geq 30 \mathrm{~min}, \geq 3$ times/week) & $31(17.5)$ & $20(16.4)$ & 0.8 & $37(20.1)$ & $14(12.2)$ & 0.076 & $24(16.6)$ & $27(17.5)$ & 0.822 \\
\hline Montreal Cognitive Assessment (adjusted) ${ }^{a}$ & $23.8 \pm 5.5$ & $23.7 \pm 5.6$ & 0.778 & $25.2 \pm 4.6$ & $21.5 \pm 6.2$ & $<0.001$ & $28.2 \pm 1.6$ & $19.5 \pm 4.6$ & $<0.001$ \\
\hline Montreal Cognitive Assessment (adjusted) ${ }^{a}<26$ & $89(50.3)$ & $65(53.3)$ & 0.61 & $75(40.8)$ & $79(68.7)$ & $<0.001$ & $0(0.0)$ & $154(100.0)$ & $<0.001$ \\
\hline Charlson Comorbidity Index & $1.1 \pm 1.1$ & $1.2 \pm 1.0$ & 0.722 & $1.2 \pm 1.0$ & $1.1 \pm 1.1$ & 0.517 & $1.1 \pm 1.0$ & $1.2 \pm 1.1$ & 0.237 \\
\hline Charlson Comorbidity Index $\geq 2$ & $48(27.1)$ & $42(34.4)$ & 0.176 & $60(32.6)$ & $30(26.1)$ & 0.232 & $42(29.0)$ & $48(31.2)$ & 0.678 \\
\hline Body mass index $\left(\mathrm{kg} / \mathrm{m}^{2}\right)$ & $24.9 \pm 3.7$ & $25.8 \pm 3.5$ & 0.039 & $25.8 \pm 3.4$ & $24.4 \pm 3.9$ & 0.002 & $25.3 \pm 3.3$ & $25.2 \pm 3.9$ & 0.930 \\
\hline \multicolumn{10}{|l|}{ ICHOM Standard Set Older Person Tier 1} \\
\hline Clinical frailty scale & $2.6 \pm 0.9$ & $2.7 \pm 1.0$ & 0.838 & $2.5 \pm 0.8$ & $3.0 \pm 1.0$ & $<0.001$ & $2.5 \pm 0.7$ & $2.8 \pm 1.1$ & $<0.001$ \\
\hline Frail & $22(12.4)$ & $13(10.7)$ & 0.639 & $13(7.1)$ & $22(19.1)$ & 0.002 & $5(3.5)$ & $30(19.5)$ & $<0.001$ \\
\hline Preferred place of death chosen & $30(17.0)$ & $13(10.7)$ & 0.128 & $29(15.8)$ & $14(12.2)$ & 0.390 & $19(13.1)$ & $24(15.6)$ & 0.541 \\
\hline Do Not Resuscitate signed & $15(8.5)$ & $6(4.9)$ & 0.237 & $17(9.2)$ & $4(3.5)$ & 0.058 & $15(10.3)$ & $6(3.9)$ & 0.029 \\
\hline \multicolumn{10}{|l|}{ ICHOM Standard Set Older Person Tier 2} \\
\hline Number of drugs & $3.4 \pm 2.6$ & $3.8 \pm 2.7$ & 0.207 & $3.2 \pm 2.6$ & $4.2 \pm 2.7$ & 0.001 & $3.5 \pm 2.7$ & $3.7 \pm 2.7$ & 0.436 \\
\hline Polypharmacy ( $\geq 5$ concurrent drugs) & $53(29.9)$ & $42(34.4)$ & 0.413 & $49(26.6)$ & $46(40.0)$ & 0.016 & $43(29.7)$ & $52(33.8)$ & 0.445 \\
\hline Number of adverse drug events & $0.0 \pm 0.2$ & $0.0 \pm 0.1$ & 0.839 & $0.0 \pm 0.2$ & $0.0 \pm 0.0$ & 0.289 & $0.0 \pm 0.2$ & $0.0 \pm 0.0$ & 0.181 \\
\hline Episodes of discomfort after medications & $0.0 \pm 0.2$ & $0.0 \pm 0.1$ & 0.599 & $0.0 \pm 0.2$ & $0.0 \pm 0.0$ & 0.103 & $0.0 \pm 0.2$ & $0.0 \pm 0.0$ & 0.103 \\
\hline Fell & $34(19.2)$ & $17(13.9)$ & 0.233 & $27(14.7)$ & $24(20.9)$ & 0.166 & $24(16.6)$ & $27(17.5)$ & 0.822 \\
\hline Number of falls & $0.3 \pm 0.6$ & $0.2 \pm 0.5$ & 0.131 & $0.2 \pm 0.6$ & $0.2 \pm 0.5$ & 0.672 & $0.2 \pm 0.6$ & $0.2 \pm 0.6$ & 0.877 \\
\hline Hospital admissions & $0.1 \pm 0.3$ & $0.3 \pm 0.6$ & $<0.001$ & $0.2 \pm 0.5$ & $0.2 \pm 0.4$ & 0.871 & $0.2 \pm 0.4$ & $0.2 \pm 0.5$ & 0.657 \\
\hline Length of hospital stay (days) & $0.5 \pm 2.6$ & $2.0 \pm 4.8$ & 0.003 & $1.0 \pm 3.5$ & $1.3 \pm 4.1$ & 0.486 & $0.9 \pm 2.7$ & $1.3 \pm 4.5$ & 0.286 \\
\hline Able to cope with own health & $162(91.5)$ & $114(93.4)$ & 0.541 & $172(93.5)$ & $104(90.4)$ & 0.337 & $138(95.2)$ & 138 (89.6) & 0.071 \\
\hline Participate in care decision-making & $163(92.1)$ & $113(92.6)$ & 0.865 & $168(91.3)$ & $108(93.9)$ & 0.410 & $135(93.1)$ & $141(91.6)$ & 0.616 \\
\hline Treated with dignity and respect & $170(96.1)$ & $119(97.5)$ & 0.48 & $178(96.7)$ & $111(96.5)$ & 0.919 & $142(97.9)$ & $147(95.5)$ & 0.234 \\
\hline Received coordinated care & $163(92.1)$ & $107(87.7)$ & 0.208 & $159(86.4)$ & $111(96.5)$ & 0.004 & $132(91.0)$ & $138(89.6)$ & 0.678 \\
\hline Discharged to place of choice & $176(99.4)$ & $120(98.4)$ & 0.36 & $183(99.5)$ & $113(98.3)$ & 0.313 & $144(99.3)$ & $152(98.7)$ & 0.597 \\
\hline Overall participation in decision-making & $4.7 \pm 0.8$ & $4.7 \pm 0.7$ & 0.863 & $4.7 \pm 0.8$ & $4.8 \pm 0.7$ & 0.353 & $4.8 \pm 0.6$ & $4.6 \pm 0.8$ & 0.175 \\
\hline High participation ( $\geq 5$ components) & $150(84.8)$ & $94(77.1)$ & 0.091 & $147(79.9)$ & $97(84.4)$ & 0.333 & $122(84.1)$ & $122(79.2)$ & 0.273 \\
\hline \multicolumn{10}{|l|}{ ICHOM Standard Set Older Person Tier 3} \\
\hline UCLA Loneliness Scale & $30.7 \pm 10.2$ & $31.3 \pm 9.8$ & 0.611 & $30.4 \pm 9.4$ & $31.8 \pm 11.0$ & 0.232 & $28.4 \pm 8.3$ & $33.4 \pm 11.0$ & $<0.001$ \\
\hline Loneliness & $46(26.0)$ & $34(27.9)$ & 0.718 & $46(25.0)$ & $34(29.6)$ & 0.386 & $22(15.2)$ & $58(37.7)$ & $<0.001$ \\
\hline Activities of daily living & $7.5 \pm 1.3$ & $7.2 \pm 1.5$ & 0.08 & $7.6 \pm 1.1$ & $7.1 \pm 1.7$ & 0.002 & $7.8 \pm 0.8$ & $7.1 \pm 1.8$ & $<0.001$ \\
\hline Any limitation of activities of daily living & $29(16.4)$ & $38(31.2)$ & 0.003 & $29(15.8)$ & $38(33.0)$ & $<0.001$ & $19(13.1)$ & $48(31.2)$ & $<0.001$ \\
\hline Walking speed (m/s) & $0.9 \pm 0.3$ & $0.9 \pm 0.3$ & 0.514 & $0.9 \pm 0.3$ & $0.8 \pm 0.3$ & $<0.001$ & $1.0 \pm 0.2$ & $0.8 \pm 0.3$ & $<0.001$ \\
\hline Slowness $(6-\mathrm{m}$ walk $<0.8 \mathrm{~m} / \mathrm{s}$ ) & $68(38.4)$ & $47(38.5)$ & 0.985 & $54(29.4)$ & $61(53.0)$ & $<0.001$ & $32(22.1)$ & $83(53.9)$ & $<0.001$ \\
\hline Moderate pain & $21(11.9)$ & $10(8.2)$ & 0.307 & $15(8.2)$ & 16 (13.9) & 0.112 & $16(11.0)$ & $15(9.7)$ & 0.714 \\
\hline
\end{tabular}


Table 3 Demographic data and ICHOM Standard Set Older Person outcome measures by sex, age, and cognitive performance status (Continued)

\begin{tabular}{|c|c|c|c|c|c|c|c|c|c|}
\hline \multirow{2}{*}{$\begin{array}{l}\text { Data values show mean } \pm \text { standard deviation or } \\
\text { number (percent) }\end{array}$} & \multicolumn{3}{|l|}{ Sex } & \multicolumn{3}{|c|}{ Age (years) } & \multicolumn{3}{|l|}{$\mathrm{MoCA}_{\mathrm{adj}}$} \\
\hline & Female & Male & $p$ & $<75$ & $\geq 75$ & $p$ & $\geq 26$ & $<26$ & p \\
\hline Depression & $25(14.1)$ & $14(11.5)$ & 0.504 & $19(10.3)$ & $20(17.4)$ & 0.078 & $16(11.0)$ & $23(14.9)$ & 0.317 \\
\hline Value-based health score & $7.3 \pm 1.9$ & $7.1 \pm 1.8$ & 0.312 & $7.6 \pm 1.6$ & $6.7 \pm 2.1$ & $<0.001$ & $7.8 \pm 1.5$ & $6.8 \pm 2.0$ & $<0.001$ \\
\hline High value-based health & $60(33.9)$ & $29(23.8)$ & 0.06 & $63(34.2)$ & $26(22.6)$ & 0.032 & $57(39.3)$ & $32(20.8)$ & 0.001 \\
\hline
\end{tabular}

${ }^{\mathrm{a} O n e}$ point added for education years $\leq 12$

ICHOM International Consortium for Health Outcomes Measurement, UCLA University of California, Los Angeles

mean plus/minus standard deviation and categorical variables as proportions. Descriptive characteristics were compared by Student $t$ test or chi-square analysis, as appropriate. To maximize statistical efficiency, the valuebased healthcare score was first treated as a continuous variable, then univariable and multivariable logistic regression analyses were used to investigate associations between corresponding variables and higher value-based healthcare status; $p<0.1$ in univariable analysis was the entry criterion for multivariable analysis.

\section{Results}

\section{Participant characteristics}

The mean value-based healthcare score of 299 enrolled participants was $7.2 \pm 1.8$ and 89 (29.8\%) had high valuebased healthcare status (Fig. 1, Table 1). Although all participants had three or more chronic conditions, the mean Charlson Comorbidity Index score was only $1.1 \pm$ 1.0 (Table 2). A minority of participants had chosen a place to die and signed Do Not Resuscitate agreements, but more than $90 \%$ reported a high level of participation in care plan decision-making. One-quarter experienced moderate loneliness and one in eight had depressed $\operatorname{mood}($ Table 1).

\section{Subgroup comparisons}

People with high versus low value-based health status were significantly younger, and cognitively intact (Fig. 1, Table 2); body mass index, alcohol consumption and exercise habits were similar between low versus high care status groups. Figure 1, Tables 1, and 3 summarize the proportions of 299 people in different ICHOM Tier categories, both overall and stratified by, value-based healthcare status (high vs low), age ( $<75$ vs $\geq 75$ years), sex, and cognitive performance ( $\mathrm{MoCA}_{\mathrm{adj}}<26 \mathrm{vs} \geq 26$ ).

\section{Linear and logistic regression analyses}

Younger age, lower Charlson Comorbidity Index score, and higher $\mathrm{MoCA}_{\mathrm{adj}}$ score independently predicted high value-based healthcare status (Table 4). For every year increase in age, the likelihood of achieving high valuebased healthcare status decreased by 5\%. (Table 5). People with higher disease burden and cognitive impairment were 60 and 51\% less likely, respectively, to attain high status (Table 5).

\section{Discussion}

This is the first study of which we know to report the value-based healthcare status of older multimorbid

Table 4 Factors associated with high-value health status in univariable and multivariate linear regression analyses

\begin{tabular}{|c|c|c|c|c|c|c|}
\hline & \multicolumn{2}{|l|}{ Univariable } & \multicolumn{4}{|l|}{ Multivariable } \\
\hline & $\beta$ coefficient & $p$ & $\beta$ coefficient $^{a}$ & $p$ & $\beta$ coefficient $^{\mathrm{b}}$ & $p$ \\
\hline Age (years) & -0.096 & $<0.001$ & -0.050 & 0.001 & -0.079 & $<0.001$ \\
\hline Male & -0.196 & 0.364 & & & & \\
\hline Education (years) & 0.080 & $<0.001$ & 0.002 & 0.921 & 0.035 & 0.110 \\
\hline Smoke tobacco & -0.480 & 0.105 & & & & \\
\hline Drink alcohol & 0.034 & 0.917 & & & & \\
\hline Exercise & 0.340 & 0.231 & 0.170 & 0.488 & 0.161 & 0.525 \\
\hline $\mathrm{CCl}$ & -0.366 & $<0.001$ & -0.297 & 0.001 & & \\
\hline $\mathrm{CCl} \geq 2$ & -0.845 & $<0.001$ & & & -0.832 & $<0.001$ \\
\hline MoCA adj & 0.155 & $<0.001$ & 0.129 & $<0.001$ & & \\
\hline MoCA $_{\text {adj }}<26$ & -0.998 & $<0.001$ & & & -0.591 & 0.005 \\
\hline Body Mass Index & 0.058 & 0.048 & 0.038 & 0.135 & 0.032 & 0.237 \\
\hline
\end{tabular}

$\mathrm{CCl}$ Charlson Comorbidity Index, $M \circ C A_{a d j}$ Montreal Cognitive Assessment adjusted (one point added for education years $\leq 12$ )

${ }^{\mathrm{a}} \mathrm{CCl}$ and $\mathrm{MoCA}_{\mathrm{adj}}$ as numerical variables

${ }^{b} \mathrm{CCl}$ and $\mathrm{MoCA}_{\mathrm{adj}}$ as categorical variables 
Table 5 Factors associated with high-value health status in univariable and multivariable logistic regression analyses

\begin{tabular}{|c|c|c|c|c|c|c|}
\hline & \multicolumn{2}{|l|}{ Univariable } & \multicolumn{4}{|l|}{ Multivariable } \\
\hline & Odds ratio $(95 \% \mathrm{Cl})$ & $p$ & Odds ratio $(95 \% \text { Cl) })^{a}$ & $p$ & Odds ratio $(95 \% \mathrm{Cl})^{\mathrm{b}}$ & $p$ \\
\hline Age (years) & $0.94(0.90,0.98)$ & 0.003 & $0.97(0.92,1.02)$ & 0.176 & $0.95(0.91,0.99)$ & 0.025 \\
\hline Male & $0.61(0.36,1.02)$ & 0.061 & $0.69(0.37,1.28)$ & 0.241 & $0.70(0.38,1.29)$ & 0.257 \\
\hline Education (years) & $1.05(0.99,1.11)$ & 0.092 & $0.99(0.93,1.06)$ & 0.783 & $1.02(0.96,1.09)$ & 0.593 \\
\hline Smoke tobacco & $0.48(0.21,1.07)$ & 0.074 & $0.57(0.23,1.42)$ & 0.228 & $0.53(0.21,1.32)$ & 0.172 \\
\hline Drink alcohol & $0.86(0.40,1.86)$ & 0.697 & & & & \\
\hline Exercise & $1.22(0.64,2.33)$ & 0.541 & & & & \\
\hline $\mathrm{CCl}$ & $0.65(0.49,0.85)$ & 0.002 & $0.66(0.49,0.89)$ & 0.006 & & \\
\hline $\mathrm{CCl} \geq 2$ & $0.40(0.22,0.74)$ & 0.004 & & & $0.40(0.21,0.76)$ & 0.005 \\
\hline MoCAadj & $1.15(1.08,1.22)$ & $<0.001$ & $1.15(1.07,1.23)$ & $<0.001$ & & \\
\hline $\mathrm{MoCA}_{\mathrm{adj}}<26$ & $0.41(0.24,0.68)$ & 0.001 & & & $0.49(0.27,0.87)$ & 0.014 \\
\hline Body Mass Index & $1.01(0.94,1.08)$ & 0.813 & & & & \\
\hline
\end{tabular}

$\mathrm{Cl}$ confidence interval, $\mathrm{CCl}$ Charlson comorbidity index, $\mathrm{MoCA}_{\text {adj }}$ Montreal Cognitive Assessment adjusted (one point added for education years $\leq 12$ )

${ }^{\mathrm{a}} \mathrm{CCl}$ and $\mathrm{MoCA}_{\mathrm{adj}}$ as numerical variables

${ }^{\mathrm{b}} \mathrm{CCl}$ and $\mathrm{MoCA} \mathrm{A}_{\text {adj }}$ as categorical variables

community-living adults. We applied the ICHOM Standard Set for Older Person to evaluate the value-based healthcare status of $\geq 65$-year-olds with multimorbidity; those who were younger, and cognitively unimpaired had higher levels of value-based healthcare status. Rates of participation in decision-making were high across all subgroups. Having high disease burden and impaired cognitive function were negatively associated with ability to achieve a high value-based healthcare score.

ICHOM Standard Set Older Person categorization into three tiers is based on Porter's health outcome hierarchy [17]. Tier 1 includes peoples' preferences for end-of-life care; choosing a place of death helps people to die at home, whereas people whose preference is unknown are more likely to be admitted to hospital for end-of-life care [18]. The proportion of Asian participants in this study expressing a preferred place of death or signing a Do Not Recuscitate Agreement was low compared with other studies [19]; this highlights an unmet need for advocacy to better prepare elderly Taiwanese people for death. The prevalence of frailty was similar to other reports $[8,20]$.

Approximately one-third of participants used $\geq 5$ concurrent medications, consistent with a study of national health insurance claims by 59,042 Taiwanese people older than 65 years [21]; nevertheless there was a low incidence of adverse drug events or discomfort after taking medications, likely due to a low rate of inappropriate medication according to insurance claims data [21]. Although people prefer more participation in decision-making and expect to be treated with dignity and respect, not all patients want to make medical decisions [22]. More than $90 \%$ of people in our study participated in decisions about their care- and received collaborative, dignified and respectful medical management, compared with $60 \%$ in a systemic review of 44 studies [22]. Although falling is usually considered a health outcome, it was chosen as a standard value-based metric because it matters to older people, their carers, and physicians. A higher rate of falls among women than men in this study was consistent with a study of 1377 community-living Taiwanese, although not statistically significant [23].

The use of SF-36 in Tier 3 to measure depression and pain has the advantage of covering many outcomes to reduce complexity, but some experts advocate considering cost-free survey tools as well [5]. The prevalence of depression in this study population was similar to previous reports from dermatology and internal medicine, and lower than among surgery patients [24]. A meta-analysis study of 19 studies reported moderate to severe chronic pain in $10-14 \%$ participants [25], which was similar to our findings. Participation and social inclusion are key components of healthy aging; $26.8 \%$ prevalence of loneliness was consistent with previous reports [26]. Although measuring physical performance is not always easy in daily practice, the ICHOM included walk speed as a Tier 3 metric because it matters to older adults [27]; the mean speed of $0.9 \mathrm{~m} / \mathrm{s}$ in this sample was much lower than reported in older healthy adults [28], reflecting that all participants were multimorbid.

The ICHOM Standard Set of outcome measures was the first tool developed for people who are older, rather than those with specific diseases or conditions. Based on findings from the study, stakeholders may devise tailormade interventions for this population and examine their effectiveness accordingly. However, the ICHOM Standard Set does not include cognitive assessment; our results show that cognitive function per se was highly 
associated with high value-based healthcare status and might be considered amended in metrics of the ICHOM Standard Set. The ICHOM variables are a combination of self-reported and professional-assessed. Most of these variables are not routinely collected, even in an integrated geriatric health care clinic. That might burden collection of data from patients and professionals. For example, a Chinese version of the 4-item screening Zarit Burden interview was not available at the time this study commenced. Complexity and burden of assessments might be a barrier to scale up use of the standard set in Taiwan or other countries, Akpan et al. argued that free tools that encompass multiple outcomes to reduce numbers of measurable variables and complexity of tools would be help for clinical and public health implementation [5].

This study had limitations. First, the ICHOM Standard Set was designed to measure longitudinal changes of valuebased health status; our cross-sectional study only presents a snapshot of baseline status. Second, convenient sampling instead of random sampling limits the representativeness and generalizability, although various dimensions studied had profiles similar to previous studies. Extrapolation of our study results to other populations may need further validation. Third, questionnaire items about falls and drug adverse events over the past year may result in recall-bias; this could be resolved by a prospective study, which is underway, and we intend to report in due course.

\section{Conclusions}

ICHOM Standard Set health outcome measures provide an opportunity to shift from a disease-centric medical paradigm to whole person care goals. The value-based health care profile in Taiwan indicates the importance of advanced age, chronic disease and cognitive impairment as barriers to achieving high value-based health status. Further longitudinal and intervention studies to examine the expedience of using ICHOM are warranted.

\section{Supplementary information}

Supplementary information accompanies this paper at https://doi.org/10. 1186/s12877-020-01734-1.

Additional file 1: Supplementary Table 1. Value-health points score components by ICHOM Standard Set outcome measures

Additional file 2: Supplementary figure 1. Flow diagram of participants recruiting process in the study.

\section{Abbreviations}

ICHOM: International Consortium for Health Outcomes Measurement; UCLA: University of California, Los Angeles; SF-36: Short-Form Health Survey; MoCA, Montreal Cognitive Assessment; MoCAadj: Montreal Cognitive Assessment adjusted by adding one point for those educated for $\leq 12$ years; CCl: Charlson Comorbidity Index

\section{Acknowledgements}

We thank all members of the project for their cooperation in data collection and management. We are indebted to all the participants for their commitment to the study. Dr. David Neil (PhD), of Full Universe Integrated Marketing, Taiwan, provided editorial assistance and his colleague Pei Chi Kuo assisted with manuscript preparation project management; their contributions were supported by funding from Taipei Veterans General Hospital.

\section{Authors' contributions}

Study concept and design, and obtaining funding: Lee WJ and Chen LK. Integrity of the data, accuracy of data analyses, and statistical expertise: Lee WJ. Acquisition of subjects/data: Lee WJ, Kao SL, Hung TS, Chang CY, Huang CF, and Tang TC. Study supervision and administrative support: Lee WJ, Peng LN, Lin CH, Lin SZ Loh CH, and Chen LK. Analysis and interpretation of data: Lee WJ, Peng LN, Lin $\mathrm{CH}$, Lin SZ, Loh CH, and Chen LK. Preparation/Critical review of the manuscript: All authors. The author(s) read and approved the final manuscript.

\section{Funding}

This study was supported by the National Health Research Institute, Taiwan (NHRI-107A1-PHCO-04181803). The sponsor has no role in the design, methods, subject recruitment, data collections, analysis and preparation of paper.

\section{Availability of data and materials}

The datasets generated and/or analysed during the current study are not publicly available due local government regulations but are available from the corresponding author on reasonable request.

\section{Ethics approval and consent to participate}

The Institutional Review Board of National Yang-Ming University approved the protocol (YM107042F). All participants provided fully informed written consent.

Consent for publication

Not applicable.

\section{Competing interests}

The authors declare that they have no competing interest.

\section{Author details}

${ }^{1}$ Aging and Health Research Center, National Yang Ming University, Taipei, Taiwan. ${ }^{2}$ Department of Geriatric Medicine, School of Medicine, National Yang Ming University, Taipei, Taiwan. ${ }^{3}$ Department of Family Medicine, Taipei Veterans General Hospital Yuanshan Branch, Yi-Lan County, Taiwan. ${ }^{4}$ Center for Geriatrics and Gerontology, Taipei Veterans General Hospital, No. 201, Sec. 2, Shih-Pai Rd, Taipei 11217, Taiwan. ${ }^{5}$ Superintendent's Office, Hualien Tzu Chi Hospital Buddhist Tzu Chi Medical Foundation, Hualien County, Taiwan. ${ }^{6}$ Center of Health and Aging, Hualien Tzu Chi Hospital Buddhist Tzu Chi Medical Foundation, Hualien County, Taiwan. ${ }^{7}$ Yingge Primary Care Center, Department of Health, New Taipei City Government, New Taipei City, Taiwan. ${ }^{8}$ Shulin Primary Care Center, Department of Health, New Taipei City Government, New Taipei City, Taiwan. 'Department of Family Medicine, National Yang-Ming university Hospital, Yi-Lan County, Taiwan. ${ }^{10}{ }^{10}$ ang's Orthopedic \& Otolaryngological Clinic's, New Taipei City, Taiwan.

Received: 30 June 2019 Accepted: 24 August 2020

Published online: 09 September 2020

References

1. Fabbri E, Zoli M, Gonzalez-Freire M, Salive ME, Studenski SA, Ferrucci L. Aging and multimorbidity: new tasks, priorities, and Frontiers for integrated Gerontological and clinical research. J Am Med Dir Assoc. 2015;16(8):640-7.

2. Rajkumar R, Conway PH, Tavenner M. CMS--engaging multiple payers in payment reform. Jama. 2014;311(19):1967-8.

3. Mendelson A, Kondo K, Damberg C, Low A, Motuapuaka M, Freeman M, O'Neil M, Relevo R, Kansagara D. The effects of pay-for-performance programs on health, health care use, and processes of care: a systematic review. Ann Intern Med. 2017;166(5):341-53.

4. Chen LK. From geriatric medicine to aging medicine. Aging Med Healthc. 2018;9(4):116-7 
5. Akpan A, Roberts C, Bandeen-Roche K, Batty B, Bausewein C, Bell D, Bramley D, Bynum J, Cameron ID, Chen LK, et al. Standard set of health outcome measures for older persons. BMC Geriatr. 2018;18(1):36.

6. Drouin $\mathrm{H}$, Walker J, McNeil H, Elliott J, Stolee P. Measured outcomes of chronic care programs for older adults: a systematic review. BMC Geriatr. 2015;15:139.

7. von Elm E, Altman DG, Egger M, Pocock SJ, Gotzsche PC, Vandenbroucke $J P$, Initiative $S$. The strengthening the reporting of observational studies in epidemiology (STROBE) statement: guidelines for reporting observational studies. Lancet. 2007;370(9596):1453-7.

8. Rockwood K, Song X, Macknight C, Bergman H, Hogan DB, McDowell I, Mitnitski A. A global clinical measure of fitness and frailty in elderly people. CMAJ. 2005;173(5):489-95.

9. Masnoon N, Shakib S, Kalisch-Ellett L, Caughey GE. What is polypharmacy? A systematic review of definitions. BMC Geriatr. 2017;17(1):230.

10. Russell DW. UCLA loneliness scale (version 3): reliability, validity, and factor structure. J Pers Assess. 1996;66(1):20-40.

11. Perry GR. Loneliness and coping among tertiary-level adult cancer patients in the home. Cancer Nurs. 1990;13(5):293-302.

12. Lawton MP, Brody EM. Assessment of older people: self-maintaining and instrumental activities of daily living. Gerontologist. 1969;9(3):179-86.

13. Chen LK, Liu LK, Woo J, Assantachai P, Auyeung TW, Bahyah KS, Chou MY, Chen LY, Hsu PS, Krairit O, et al. Sarcopenia in Asia: consensus report of the Asian working Group for Sarcopenia. J Am Med Dir Assoc. 2014;15(2):95-101.

14. Matcham F, Norton S, Steer S, Hotopf M. Usefulness of the SF-36 health survey in screening for depressive and anxiety disorders in rheumatoid arthritis. BMC Musculoskelet Disord. 2016;17:224.

15. Tsai CF, Lee WJ, Wang SJ, Shia BC, Nasreddine Z, Fuh JL. Psychometrics of the Montreal cognitive assessment (MoCA) and its subscales: validation of the Taiwanese version of the MoCA and an item response theory analysis. Int Psychogeriatr. 2012;24(4):651-8.

16. Charlson ME, Charlson RE, Peterson JC, Marinopoulos SS, Briggs WM, Hollenberg JP. The Charlson comorbidity index is adapted to predict costs of chronic disease in primary care patients. J Clin Epidemiol. 2008;61(12):1234-40.

17. Porter ME. What is value in health care? N Engl J Med. 2010;363(26):2477-81.

18. Ali M, Capel M, Jones G, Gazi T. The importance of identifying preferred place of death. BMJ Support Palliat Care. 2019;9(1):84-91. https://doi.org/10. 1136/bmjspcare-2015-000878.

19. Cook I, Kirkup AL, Langham L, Malik MA, Marlow G, Sammy I. End of life care and do not resuscitate orders: how much does age influence decision making? A Systematic Review and Meta-Analysis. Gerontol Geriatr Med. 2017;3:2333721417713422.

20. Lee WJ, Peng LN, Lin CH, Lin HP, Loh CH, Chen LK. The synergic effects of frailty on disability associated with urbanization, multimorbidity, and mental health: implications for public health and medical care. Sci Rep. 2018;8(1):14125.

21. Lu WH, Wen YW, Chen LK, Hsiao FY. Effect of polypharmacy, potentially inappropriate medications and anticholinergic burden on clinical outcomes: a retrospective cohort study. CMAJ. 2015;187(4):E130-7.

22. Brom L, Hopmans W, Pasman HR, Timmermans DR, Widdershoven GA Onwuteaka-Philipsen BD. Congruence between patients' preferred and perceived participation in medical decision-making: a review of the literature. BMC Med Inform Decis Mak. 2014;14:25.

23. Lin CH, Liao KC, Pu SJ, Chen YC, Liu MS. Associated factors for falls among the community-dwelling older people assessed by annual geriatric health examinations. PLoS One. 2011;6(4):e18976.

24. Wang J, Wu X, Lai W, Long E, Zhang X, Li W, Zhu Y, Chen C, Zhong X, Liu Z, et al. Prevalence of depression and depressive symptoms among outpatients: a systematic review and meta-analysis. BMJ Open. 2017;7(8):e017173.

25. Fayaz A, Croft P, Langford RM, Donaldson L, Jones GT. Prevalence of chronic pain in the UK: a systematic review and meta-analysis of population studies. BMJ Open. 2016;6(6):e010364.

26. Cantarero-Prieto D, Pascual-Saez M, Blazquez-Fernandez C. Social isolation and multiple chronic diseases after age 50: a European macro-regional analysis. PLoS One. 2018;13(10):e0205062.

27. Studensk S, Perera S, Patel $K$, Rosano C, Faulkner $K$, Inżtani M, Brach J, Chandler J, Cawthon P, Connor EB, et al. Gait speed and survival in older adults. Jama. 2011:305(1):50-8.

28. Liu LK, Lee WJ, Liu CL, Chen LY, Lin MH, Peng LN, Chen LK. Age-related skeletal muscle mass loss and physical performance in Taiwan: implications to diagnostic strategy of sarcopenia in Asia. Geriatr Gerontol Int. 2013;13(4):964-71.

\section{Publisher's Note}

Springer Nature remains neutral with regard to jurisdictional claims in published maps and institutional affiliations.

Ready to submit your research? Choose BMC and benefit from:

- fast, convenient online submission

- thorough peer review by experienced researchers in your field

- rapid publication on acceptance

- support for research data, including large and complex data types

- gold Open Access which fosters wider collaboration and increased citations

- maximum visibility for your research: over $100 \mathrm{M}$ website views per year

At $\mathrm{BMC}$, research is always in progress.

Learn more biomedcentral.com/submissions 